\title{
MATURAÇÃO DE GRAVIOLAS APÓS ARMAZENAMENTO EM ATMOSFERA CONTROLADA
}

\author{
Alex Guimarães Sanches ${ }^{1}$, Maryelle Barros da Silva ${ }^{2}$, Elaine Gleice Silva Moreira ${ }^{3}$, Jaqueline Macedo Costa ${ }^{1}$, \\ Carlos Alberto Martins Cordeiro ${ }^{1}$ \\ ${ }^{1}$ Universidade Federal do Pará - UFPA, Belém, PA. ${ }^{2}$ SEBRAE, Marabá, PA. ${ }^{3}$ SEBRAE, Altamira, PA
}

\section{RESUMO}

Por se tratar de um fruto climatérico a graviola apresenta vida útil relativamente curta principalmente pela perda de firmeza provocada pelo amadurecimento comprometendo assim sua expansão para mercados distantes. Diante disso, o presente trabalho tem por objetivo avaliar o efeito de diferentes condições de atmosfera controlada no prolongamento da vida útil deste fruto. O delineamento experimental adotado foi inteiramente casualizado em arranjo fatorial $5 \times 3$ sendo cinco variações de gás carbônico $\left(\mathrm{CO}_{2}\right)$ sob atmosfera controlada e três tempos de armazenamento após a saída das câmaras, com cinco repetições e a parcela experimental composta por dois frutos. Após quinze dias em atmosfera controlada e mais oito dias em temperatura ambiente avaliou-se: coloração da casca e da polpa, produção de etileno, taxa respiratória, firmeza, acidez titulável, pH, sólidos solúveis, teor de amido, incidência de podridões e degenerescência da polpa. As pressões maiores de $\mathrm{CO}_{2}$ comprometeram a qualidade dos frutos por acelerar a síntese de etileno favorecendo a perda de firmeza, a degradação dos compostos clorofílicos da casca e da polpa além de reduzir a qualidade após quatro dias de armazenamento em condições ambiente. As menores pressões de $\mathrm{CO}_{2}$ mostraram-se mais eficientes para o armazenamento e prolongamento da vida útil deste fruto.

Palavras-chave: Annona muricata L.; distúrbios fisiológicos; etileno, pós-colheita.

\section{MATURATION GRAVIOLAS AFTER STORAGE IN CONTROLLED ATMOSPHERE}

\begin{abstract}
For it is a climacteric fruit sour sop has relatively short shelf life mainly due to the loss of firmness caused by maturation thus compromising its expansion into distant markets. Thus, this study aims to evaluate the effect of different controlled atmosphere conditions in extending the life of the fruit. The experimental design was completely randomized in a $5 \times 3$ factorial arrangement with five variations of carbon dioxide (CO2) under controlled atmosphere storage and three times after leaving the chambers, with five replicates and the experimental plot was composed of two fruits. After fifteen days in a controlled atmosphere and eight days, at room temperature were evaluated: color of skin and pulp, ethylene production, respiration rate, firmness, titratable acidity, $\mathrm{pH}$, soluble solids, starch content, incidence of decay and degeneracy pulp. The greatest pressures of $\mathrm{CO}_{2}$ committed, fruit quality by accelerating ethylene synthesis favoring the loss of firmness, degradation of chlorophyll compounds of the skin and pulp and reduces the quality after four days of storage at ambient conditions. The lower pressure $\mathrm{CO} 2$ were more efficient for storing and extending the life of the fruit.
\end{abstract}

Keywords: Annona muricata L.; physiological disorders; ethylene; postharvest.

\section{INTRODUÇÃO}

A gravioleira é uma frutífera tropical da família Annonaceae, que tem origem na América Central e norte da América do Sul. É um fruto bastante procurado devido à suas qualidades sensoriais e funcionais (ZACARONI et al., 2014).

A alta perecibilidade da graviola após a colheita caracterizada pelo amaciamento da polpa 
é um dos maiores entraves à comercialização da fruta fresca principalmente por conta da distância dos centros de produção aos mercados consumidores, ocasionando desta forma relevantes perdas econômicas (FREITAS et al., 2013).

O emprego de tecnologias que retardem o amadurecimento e aumentem a vida útil póscolheita é de grande necessidade. Dentre essas tecnologias destaca-se o uso do armazenamento em atmosfera controlada. Neste sistema, além da temperatura, são também controladas as pressões parciais de oxigênio $\mathrm{O}_{2}$, gás carbônico $\mathrm{CO}_{2}$ e a umidade relativa do ar (UR) durante o armazenamento de modo que a baixa pressão parcial de $\mathrm{O}_{2}$ e alta de $\mathrm{CO}_{2}$, reduzem a produção de etileno e a respiração. Por meio da atmosfera controlada, alteram-se também outros processos dependentes do etileno e da respiração, como por exemplo, a perda da firmeza da polpa, o consumo de ácidos e a coloração (GUPTA et al., 2009).

O prolongamento da vida de prateleira dos frutos após o armazenamento em atmosfera controlada e com diferentes variações de $\mathrm{CO}_{2}$ tem sido comprovado por diversos autores em pesquisas com Ameixas (STEFFENS et al., 2013) e morangos (CUNHA JÚNIOR et al., 2012)

As condições de atmosfera controlada sobre a variação da concentração de gás carbônico $\left(\mathrm{CO}_{2}\right)$ que melhor mantém a qualidade da graviola ainda não estão estabelecidas e levando-se em consideração que cada espécie apresenta um metabolismo diferenciado, o que repercute no armazenamento, é de extrema importância o conhecimento dos níveis de gases mais adequados para a conservação da qualidade dos frutos desta frutífera.

Diante do exposto, a presente pesquisa tem por objetivo avaliar o efeito de diferentes variações de gás carbônico $\left(\mathrm{CO}_{2}\right)$ durante o armazenamento de graviolas variedade "Morada" em atmosfera controlada com o intuito de retardar seu amadurecimento e prolongar sua vida de prateleira, fazendo que a mesma alcance mercados mais distantes.

\section{MATERIAL E MÉTODOS}

Graviolas da variedade "Morada" foram colhidas de plantas originadas de mudas do tipo pé franco com idade médias de seis anos, em estádio de maturação fisiológica alcançada na cor verde claro com peso médio variando entre $800 \mathrm{~g}$ a 1,0 $\mathrm{kg}$. A colheita foi realizada no Centro Integrado de Fruticultura da Transamazônica localizado no município de Uruará-PA, levando-se em consideração frutos ausentes de ataques por pragas e doenças e de defeitos de formação. A propriedade está localizada sob as coordenadas $03^{\circ}$ $42^{\prime} 54^{\prime \prime}$ de latitude Sul e $53^{\circ} 44^{\prime} 24^{\prime \prime}$ de longitude a Oeste e possui uma área plantada em torno de 10 hectares com graviola.

Imediatamente após a colheita os frutos foram envolvidos com jornal e acomodados em caixas térmicas sendo então transportados por aproximadamente 1 hora até o laboratório de Tecnologia Pós-colheita da Universidade Federal do Pará, distante $60 \mathrm{~km}$ do centro de produção. No laboratório os frutos foram sanitizados em água clorada a $5 \%$ por um período de cinco minutos seguido de enxague em água corrente para a retirada do excesso do sanitizante.

Os frutos foram secos a temperatura ambiente e em seguida armazenados em câmaras de armazenamento com fluxo contínuo de mistura gasosa de $100 \mathrm{~mL} /$ minuto, contendo concentrações de oxigênio e gás carbônico. As câmaras tinham capacidade de $300 \mathrm{~L}$ e a temperatura de armazenamento foi de $5^{\circ} \mathrm{C}$ e $90 \%$ de UR nas seguintes condições de atmosfera modificada: $\mathrm{T} 1=$ $2,0 \mathrm{kPa}$ de $\mathrm{O}_{2}+<0,05 \mathrm{kPa}$ de $\mathrm{CO}_{2}$ representando o tratamento (controle); $\mathrm{T} 2=2,0 \mathrm{kPa}$ de $\mathrm{O}_{2}+<1,0 \mathrm{kPa}$ de $\mathrm{CO}_{2} ; \mathrm{T} 3=2,0 \mathrm{kPa}$ de $\mathrm{O}_{2}+2,0 \mathrm{kPa}$ de $\mathrm{CO}_{2} ; \mathrm{T} 4=2,0$ $\mathrm{kPa}$ de $\mathrm{O}_{2}+2,5 \mathrm{kPa}$ de $\mathrm{CO}_{2} ; \mathrm{T} 5=2,0 \mathrm{kPa}$ de $\mathrm{O}_{2}+3,0$ $\mathrm{kPa}$ de $\mathrm{CO}_{2}$.

O delineamento experimental adotado foi - DIC inteiramente casualizado sobre arranjo fatorial $5 \times 3$ sendo: cinco condições de atmosfera controlada com variação na concentração de gás carbônico $\left(\mathrm{CO}_{2}\right)$ e três tempos de armazenamento após saída das câmaras: 0,4 e 8 dias. Cada tratamento era formado por cinco repetições e a parcela experimental constituída por dois frutos.

Antes da entrada dos frutos nas câmaras um lote formado por oito frutos foi avaliado quanto à qualidade apresentando valores médios de 5,36, de $\mathrm{pH}$, acidez de 0,19 g/100 g ácido cítrico, sólidos solúveis de 7,22 e índice de amido de 10,86 $\mathrm{g} / 100 \mathrm{~g}^{-1}$.

As pressões parciais de $\mathrm{CO}_{2}$ foram obtidas por meio da injeção deste gás nas câmaras até as pressões desejadas. 0 monitoramento das 
temperaturas foi feito diariamente, utilizando termômetros de mercúrio.

Para a manutenção constante dos níveis de $\mathrm{O}_{2}$ e $\mathrm{CO}_{2}$, estes foram monitorados e corrigidos diariamente. O monitoramento foi feito com um sistema de controle automático de gases da marca Kronenberger-Climasul. $\mathrm{O}_{2} \mathrm{O}_{2}$ consumido pela respiração foi reposto através da injeção de ar nas câmaras. $\mathrm{O} \mathrm{CO}_{2}$ em excesso foi absorvido por uma solução de hidróxido de potássio (40\%), através da qual foram circulados os gases das câmaras. Após 15 dias de armazenamento, os frutos foram avaliados nos tempos: 0 (saída da câmara), 4 e 8 dias em condições ambiente $\left(23^{\circ} \mathrm{C}\right.$ e $85 \%$ de UR) simulando a comercialização quanto a coloração da casca e da polpa sendo determinada com colorímetro Minolta, modelo CR- 300. Foram tomadas duas leituras por fruta, em lados opostos de sua região equatorial e os resultados expressos em ângulo de cor $\left(h^{\circ}\right)$ para coloração da casca e para a coloração da polpa os resultados foram expressos em ângulo de luminosidade " $L$ ", onde quanto menor o valor de " $L$ " mais escurecida estaria a polpa, conforme AOAC (2008).

A taxa respiratória e a produção de etileno foram determinadas por cromatografia gasosa em cromatógrafo Varian 3300. As amostras de cada repetição foram colocadas em minicâmaras hermeticamente fechadas sendo calculado o gás do espaço livre dos recipientes através de analisadores eletrônicos de $\mathrm{CO}_{2}$ e $\mathrm{C}_{2} \mathrm{H}_{4}$, marca Agri-Datalog. Com base na concentração de $\mathrm{CO}_{2}$, no volume do espaço livre, na massa de frutos e no tempo de fechamento, foi calculada a respiração e a produção de etileno sendo os valores expressos em mililitro de $\mathrm{CO}_{2}$ kg-1 $\mathrm{h}^{-1}$ e $\mathrm{nmol} \mathrm{C}_{2} \mathrm{H}_{4} \mathrm{~kg}^{-1}$. $\mathrm{h}^{-1}$, respectivamente.

Firmeza $(\mathrm{N})$, determinada com o auxílio de um penetrômetro Mc Cormick, modelo FT 327. Foram feitas quatro medições na região equatorial dos frutos.

Após oito dias em condições ambientes os frutos foram avaliados quanto ao teor de sólidos solúveis, obtido por leitura direta em refratômetro marca Atago, modelo Pallete - 101 e os resultados expressos em ${ }^{\circ}$ Brix.

A determinação da acidez titulável foi feita de acordo com a metodologia indicada pela AOAC (2008) e os resultados expressos em porcentagem de ácido cítrico na polpa. As análises de $\mathrm{pH}$ foram realizadas com um potenciômetro eletrônico, utilizando-se cinco gramas de polpa diluída em 50 $\mathrm{mL}$ de água destilada.

A incidência de podridões foi avaliada pela contagem dos frutos afetados que apresentaram lesões externas maiores do que $5 \mathrm{~mm}$ de diâmetro com características de infecção por patógenos seguindo o proposto por AOAC (2008). Os resultados foram expressos em percentagem (\%).

O teor de amido foi realizado por hidrólise ácida, conforme AOAC (2008). A partir do filtrado obtido, determinou-se o conteúdo de açúcares redutores, por meio do ácido 3,5-dinitrosalicílico (DNS) com a multiplicação dos resultados pelo fator 0,9 , para se obter o rendimento da transformação da sacarose em glicose, na hidrólise e os resultados expressos em $\mathrm{g} / 100 \mathrm{~g}^{-1}$.

A percentagem de incidência de degenerescência da polpa foi avaliada por meio de um corte na secção transversal dos frutos e de contagem dos frutos que apresentavam qualquer tipo de escurecimento, aspecto úmido e pequenas cavidades, nas regiões internas da polpa, segundo AOAC (2008).

Os dados foram submetidos a análise de variância e a comparação das médias pelo teste de Tukey a $5 \%$ de probabilidade utilizando o software estatístico Assistat 7.7 versão beta. A descrição das variáveis, em função dos períodos de armazenamento, foi feita utilizando-se a análise de regressão e os modelos foram selecionados observando a significância do teste $F$ para cada modelo e seus respectivos coeficientes de determinação.

\section{RESULTADOS E DISCUSSÃO}

A coloração da casca e da polpa foi influenciada pelas condições de atmosfera controlada principalmente quando expostas a temperatura ambiente (Tabela 1). Após a saída das câmaras e ao fim de quatro dias em condições ambiente os frutos mantidos nas pressões de 2,0 $\mathrm{kPa}$ de $\mathrm{O}_{2}+3,0 \mathrm{kPa}$ de $\mathrm{CO}_{2}$ apresentaram maior variação na cor da casca apresentando uma tonalidade mais amarelada quando comparada as demais condições de atmosfera controlada. A condição de $2,0 \mathrm{kPa}$ de $\mathrm{O}_{2}+<0,05 \mathrm{kPa}$ de $\mathrm{CO}_{2}$ favoreceu uma coloração mais verde aos frutos na saída da câmara diferindo significativamente dos demais, contudo ao quarto dia não apresentou 
variação estatística quando comparado aos frutos mantidos sob 2,0 kPa de $\mathrm{O}_{2}+<1,0 \mathrm{kPa}$ de $\mathrm{CO}_{2}$, e 2,0 $\mathrm{kPa}$ de $\mathrm{O}_{2}+2,0 \mathrm{kPa}$ de $\mathrm{CO}_{2}$. Após oito dias sob condições ambiente, não houve diferença na cor da casca dos frutos entre os tratamentos.

No que se refere a coloração da polpa $(L)$, observou-se que os frutos armazenados sob 2,0 $\mathrm{kPa}$ de $\mathrm{O}_{2}+<0,05 \mathrm{kPa}$ de $\mathrm{CO}_{2}$ apresentaram tonalidade mais clara em relação aos demais, principalmente quando houve incremento na concentração de $\mathrm{CO}_{2}\left(2,0 \mathrm{kPa}\right.$ de $\mathrm{O}_{2}+2,5 \mathrm{kPa}$ de $\mathrm{CO}_{2}$ e $2,0 \mathrm{kPa}$ de $\mathrm{O}_{2}+3,0 \mathrm{kPa}$ de $\mathrm{CO}_{2}$ ). Ao longo do tempo de armazenamento em temperatura ambiente notou-se interação significativa entre as condições de $A C$ avaliadas (Tabela 1).

A redução nos valores da coloração da casca $\left(h^{\circ}\right)$ e da polpa $(L)$ quando mantidos em maiores pressões de $\mathrm{CO}_{2}$ verificados neste experimento corroboram com os estudos de Pedreschi et al., (2009) que concentrações mais elevadas de $\mathrm{CO}_{2}$ durante $\mathrm{o}$ armazenamento reduzem a atividade respiratória dos frutos ocasionando a formação de produtos tóxicos ás células que formam a clorofila. Franck et al. (2007) em análise sob atmosfera controlada de frutos definiu maior escurecimento da polpa quando há o aumento na pressão de $\mathrm{CO}_{2}$.

Cunha Junior et al. (2012) avaliando morangos da variedade "Oso grande" notaram maior perda no ângulo de cor da casca e da polpa quando houve incremento nas concentrações de $\mathrm{CO}_{2}$.

Tabela 1. Valores médios sobre a coloração da casca $\left(h^{\circ}\right)$ e coloração da polpa $(L)$ em graviolas armazenadas sob diferentes variações de gás carbônico $\left(\mathrm{CO}_{2}\right)$ em atmosfera controlada por quinze dias com posterior armazenamento em condições de temperatura ambiente por oito dias.

Condições de Atmosfera Controlada

\begin{tabular}{|c|c|c|c|}
\hline & \multicolumn{3}{|c|}{ Coloração da Casca $\left(h^{\circ}\right)$} \\
\hline Controle $\left.(2,0 \mathrm{kPa} \mathrm{O}+<0,05 \mathrm{kPa} \mathrm{CO})_{2}\right)$ & 44,5 aA & 37,3 aA & 37,9 aA \\
\hline $2,0 \mathrm{kPaO}_{2}+<1,0 \mathrm{kPa} \mathrm{CO} 2$ & $42,6 \mathrm{bA}$ & 36,9 aA & $32,2 \mathrm{aA}$ \\
\hline $2,0 \mathrm{kPaO}_{2}+2,0 \mathrm{kPaCO}$ & $42,9 \mathrm{bA}$ & 36,4 aA & 33,4 aA \\
\hline $2,0 \mathrm{kPaO} \mathrm{O}_{2}+2,5 \mathrm{kPaCO}$ & $41,5 \mathrm{bA}$ & $34,5 \mathrm{bB}$ & $30,4 \mathrm{aB}$ \\
\hline $2,0 \mathrm{kPa} \mathrm{O}+3,0 \mathrm{kPaCO}$ & $38,8 \mathrm{cA}$ & $32,4 \mathrm{cB}$ & $26,4 \mathrm{aB}$ \\
\hline \multirow[t]{2}{*}{$\mathrm{CV}=(\%)$} & 11,83 & 12,13 & 11,91 \\
\hline & \multicolumn{3}{|c|}{ Coloração da Polpa $(L)$} \\
\hline Controle $\left(2,0 \mathrm{kPa} \mathrm{O}{ }_{2}+<0,05 \mathrm{kPa} \mathrm{CO}\right)_{2}$ & 88,3 aA & $85,4 \mathrm{aA}$ & 80,3 aA \\
\hline $2,0 \mathrm{kPa} \mathrm{O}_{2}+<1,0 \mathrm{kPa} \mathrm{CO}$ & 87,5 aA & $82,3 \mathrm{abA}$ & $77,5 \mathrm{bA}$ \\
\hline $2,0 \mathrm{kPaO}_{2}+2,0 \mathrm{kPa} \mathrm{CO} 2_{2}$ & $84,8 \mathrm{bA}$ & $79,8 \mathrm{bA}$ & 75,9 bcA \\
\hline $2,0 \mathrm{kPaO}{ }_{2}+2,5 \mathrm{kPaCO}_{2}$ & 84,2 bA & $79,1 \mathrm{bA}$ & $71,6 \mathrm{cB}$ \\
\hline $2,0 \mathrm{kPa} \mathrm{O}+3,0 \mathrm{kPaCO}$ & $82,7 \mathrm{cA}$ & $79,3 \mathrm{bA}$ & $70,8 \mathrm{cB}$ \\
\hline$C V=(\%)$ & 11,55 & 11,93 & 10,87 \\
\hline
\end{tabular}

Médias seguidas pela mesma letra na coluna (minúsculas) e na linha (maiúsculas) diferem entre si. Foi aplicado o teste de Tukey ao nível de $5 \%$ de probabilidade. ns: não significativo.

A taxa de produção de etileno apresentou variação estatística entre as diferentes condições de $\mathrm{AC}$ e ao longo do tempo de armazenamento em temperatura ambiente (Tabela 2). No tempo zero (saída da câmara) o nível médio de etileno foi maior nos frutos mantidos sob as pressões de 2,0 $\mathrm{kPa}$ de $\mathrm{O}_{2}+<1,0 \mathrm{kPa}$ de $\mathrm{CO}_{2}$ e $2,0 \mathrm{kPa}$ de $\mathrm{O}_{2}+2,0$ $\mathrm{kPa}$ de $\mathrm{CO}_{2}$ quando comparado aos demais. Ao fim de quatro e oito dias em condição ambiente as pressões de $\mathrm{AC}$ a $2,0 \mathrm{kPa}$ de $\mathrm{O}_{2}+2,0 \mathrm{kPa}$ de $\mathrm{CO}_{2}$, $2,0 \mathrm{kPa}$ de $\mathrm{O}_{2}+2,5 \mathrm{kPa}$ de $\mathrm{CO}_{2}$ e 2,0 $\mathrm{kPa}$ de $\mathrm{O}_{2}+3,0$ $\mathrm{kPa}$ de $\mathrm{CO}_{2}$ apresentaram as maiores taxas diferindo estatisticamente dos demais. A condição de $2,0 \mathrm{kPa}$ de $\mathrm{O}_{2}+<0,05 \mathrm{kPa}$ de $\mathrm{CO}_{2}$ evidenciou as menores evoluções não diferindo nos dias 0 e 4 , contudo ao oitavo dia a pressão de $2,0 \mathrm{kPa}$ de $\mathrm{O}_{2}+$ $<1,0 \mathrm{kPa}$ de $\mathrm{CO}_{2}$ e $, 0,05 \mathrm{kPa}$ de $\mathrm{CO}_{2}$ foram menores (Tabela 2). 
A baixa produção de etileno observada na condição de $1,0 \mathrm{kPa}$ de $\mathrm{O}_{2}+<0,05 \mathrm{kPa}$ de $\mathrm{CO}_{2}$ pode estar relacionada a quebra da enzima ACC oxidase que necessita da presença de $\mathrm{CO}_{2}$ para acelerar sua atuação e consequentemente o amadurecimento (BOTH et al., 2014).

Tabela 2. Valores médios sobre a produção de etileno $\left(\eta m o l ~ C_{2} \mathrm{H}_{4} \mathrm{~kg}^{-1} \cdot \mathrm{h}^{-1}\right.$ ) em graviolas armazenadas sob diferentes variações de gás carbônico $\left(\mathrm{CO}_{2}\right)$ em atmosfera controlada por quinze dias com posterior armazenamento em condições de temperatura ambiente por oito dias.

\begin{tabular}{|c|c|c|c|}
\hline \multirow{2}{*}{ Condições de Atmosfera Controlada } & \multicolumn{3}{|c|}{ Dias de armazenamento em temperatura ambiente } \\
\hline & 0 (saída da câmara) & 4 & 8 \\
\hline & \multicolumn{3}{|c|}{ Produção de etileno (nmol C2H4 kg-1. h-1) } \\
\hline Controle $\left(2,0 \mathrm{kPa} \mathrm{O}_{2}+<0,05 \mathrm{kPa} \mathrm{CO}\right)_{2}$ & $110,6 \mathrm{cA}$ & $157,4 \mathrm{cB}$ & 298,9 bC \\
\hline $2,0 \mathrm{kPa} \mathrm{O}{ }_{2}+<1,0 \mathrm{kPa} \mathrm{CO} 2$ & $131,6 \mathrm{aB}$ & $172,8 \mathrm{bBC}$ & 304,4 bCD \\
\hline $2,0 \mathrm{kPaO} \mathrm{O}_{2}+2,0 \mathrm{kPa} \mathrm{CO}$ & $133,4 \mathrm{aB}$ & $184,2 \mathrm{abC}$ & $334,5 \mathrm{aD}$ \\
\hline $2,0 \mathrm{kPaO}{ }_{2}+2,5 \mathrm{kPaCO}$ & $121,8 \mathrm{bAB}$ & $193,4 \mathrm{aC}$ & $336,8 \mathrm{aD}$ \\
\hline $2,0 \mathrm{kPaO} \mathrm{O}_{2}+3,0 \mathrm{kPaCO}$ & $123,9 \mathrm{bAB}$ & $199,2 \mathrm{aC}$ & $337,7 \mathrm{aD}$ \\
\hline$C V=(\%)$ & 11,31 & 9,93 & 13,56 \\
\hline
\end{tabular}

Médias seguidas pela mesma letra na coluna (minúsculas) e na linha (maiúsculas) diferem entre si. Foi aplicado o teste de Tukey ao nível de $5 \%$ de probabilidade. ns: não significativo.

De acordo com a Tabela 3, notou-se que a taxa respiratória dos frutos foi significativamente afetada pelas condições de AC. Na saída das câmaras, observou-se que aqueles armazenados sob 2,0 $\mathrm{kPa}$ de $\mathrm{O}_{2}+2,5 \mathrm{kPa}$ de $\mathrm{CO}_{2}$ e $2,0 \mathrm{kPa}$ de $\mathrm{O}_{2}+$ $3,0 \mathrm{kPa}$ de $\mathrm{CO}_{2}$ apresentaram as maiores médias em relação aqueles mantidos sob baixa pressão de $\mathrm{CO}_{2}\left(2,0 \mathrm{kPa}\right.$ de $\mathrm{O}_{2}+<0,05 \mathrm{kPa}$ de $\mathrm{CO}_{2}$ e $2,0 \mathrm{kPa}$ de $\mathrm{O}_{2}+<1,0 \mathrm{kPa}$ de $\mathrm{CO}_{2}$ ) que apresentaram médias em torno de 64,3 e $66,5 \mathrm{CO}_{2} \mathrm{~kg}-1 \mathrm{~h}^{-1}$, respectivamente. Após quatro e oito dias em temperatura ambiente notou-se uma inversão quanto ao padrão respiratório dos frutos em relação a $A C$, deste modo, as menores pressões de $\mathrm{O}_{2}\left(2,0 \mathrm{kPa}\right.$ de $\mathrm{O}_{2}+$ $<0,05 \mathrm{kPa}$ de $\mathrm{CO}_{2}$ e $2,0 \mathrm{kPa}$ de $\mathrm{O}_{2}+<1,0 \mathrm{kPa}$ de $\mathrm{CO}_{2}$ ), passaram a apresentar as maiores médias estendendo-se até o fim de oito dias.
Ao fim de oito dias de armazenamento observou-se que o aumento das pressões de $\mathrm{CO}_{2}$ representado pelos tratamentos $\left(2,0 \mathrm{kPa}\right.$ de $\mathrm{O}_{2}+$ 2,5 $\mathrm{kPa}$ de $\mathrm{CO}_{2}$ e 2,0 $\mathrm{kPa}$ de $\mathrm{O}_{2}+3,0 \mathrm{kPa}$ de $\mathrm{CO}_{2}$ ), garantiram as menores taxas respiratórias, as quais não diferiram entre si e foram superiores as demais condições de AC (Tabela 3).

Provavelmente a menor taxa respiratória observada nos frutos sob altas pressões de $\mathrm{CO}_{2}$ pode estar relacionada ao efeito inibitório que esse gás exerce sobre as enzimas responsáveis pela síntese de maturação como a fosfofrutoquinase, succinato desidrogenase e isocitrato desidrogenase (SUGAR; BASILE, 2013).

Tabela 3. Valores médios sobre a taxa respiratória $\left(\mathrm{CO}_{2} \mathrm{~kg}-1 \mathrm{~h}^{-1}\right)$ em graviolas armazenadas sob diferentes variações de gás carbônico $\left(\mathrm{CO}_{2}\right)$ em atmosfera controlada por quinze dias com posterior armazenamento em condições de temperatura ambiente por oito dias.

Condições de Atmosfera Controlada

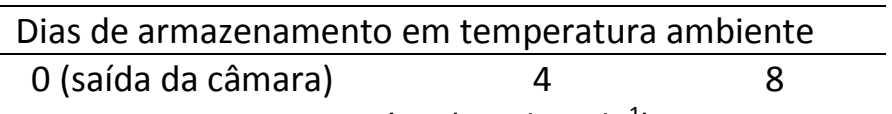

Taxa respiratória $\left(\mathrm{CO}_{2} \mathrm{~kg}-1 \mathrm{~h}^{-1}\right)$ 
Controle $\left(2,0 \mathrm{kPa} \mathrm{O}{ }_{2}+<0,05 \mathrm{kPa} \mathrm{CO}\right)_{2}$

$2,0 \mathrm{kPaO}{ }_{2}+<1,0 \mathrm{kPaCO}$

$2,0 \mathrm{kPaO}{ }_{2}+2,0 \mathrm{kPaCO}$

$2,0 \mathrm{kPaO}_{2}+2,5 \mathrm{kPaCO}$

$2,0 \mathrm{kPaO}_{2}+3,0 \mathrm{kPaCO}$
$64,3 \mathrm{cA}$

$66,5 \mathrm{cA}$

$68,5 \mathrm{bA}$

70,7 aA

71,3 aA

$$
\mathrm{CV}=(\%)
$$

13,89

$78,6 \mathrm{aB}$

$79,4 \mathrm{aB}$

$75,1 \mathrm{bB}$

$74,9 \mathrm{bA}$

$74,8 \mathrm{bA}$
86,4 aC

$85,6 \mathrm{aC}$

$80,5 \mathrm{bB}$

$79,8 \mathrm{bB}$

$79,2 \mathrm{bB}$

Médias seguidas pela mesma letra na vertical não diferem entre si. Foi aplicado o teste de tukey ao nível de $5 \%$ de probabilidade.

No que tange a firmeza dos frutos, observou-se que na saída da câmara os frutos mantidos na pressão de $2,0 \mathrm{kPa}$ de $\mathrm{O}_{2}+<1,0 \mathrm{kPa}$ de $\mathrm{CO}_{2}$ apresentaram-se mais firmes, contudo não apresentaram diferença significativa quando comparada a condição de $2,0 \mathrm{kPa}$ de $\mathrm{O}_{2}+<0,05 \mathrm{kPa}$ de $\mathrm{CO}_{2}$ e 2,0 $\mathrm{kPaO} \mathrm{O}_{2}+2,0 \mathrm{kPa} \mathrm{CO}$ (Tabela 4).

Após quatro dias, a firmeza não foi afetada pelas condições de $\mathrm{O}_{2}$ e $\mathrm{CO}_{2}$. Ao final de oito dias as médias entre os tratamentos com $2,0 \mathrm{kPa}$ de $\mathrm{O}_{2}+$ $2,5 \mathrm{kPa}$ de $\mathrm{CO}_{2}$ e $2,0 \mathrm{kPa}$ de $\mathrm{O}_{2}+3,0 \mathrm{kPa}$ de $\mathrm{CO}_{2}$ não diferiram entre si e revelaram um elevado nível de amolecimento dos frutos com médias de $6,54 \mathrm{~N}$ e $6,21 \mathrm{~N}$ respectivamente (Tabela 4). Essa redução mais acentuada na firmeza dos frutos mantidos em altas pressões de $\mathrm{CO}_{2}$ está relacionada ao aumento da produção de etileno, verificados na Tabela 2 , uma vez que a síntese deste gás ativa enzimas que degradam componentes da parede celular responsáveis pelas modificações na textura dos frutos (PAYASI et al., 2009).

As condições de $2,0 \mathrm{kPa}$ de $\mathrm{O}_{2}+<0,05 \mathrm{kPa}$ de $\mathrm{CO}_{2} 2,0 \mathrm{kPa}$ de $\mathrm{O}_{2}+<1,0 \mathrm{kPa}$ de $\mathrm{CO}_{2}$ e $2,0 \mathrm{kPa}$ de $\mathrm{O}_{2}+2,0 \mathrm{kPa}$ de $\mathrm{CO}_{2}$ não diferiram ao fim do armazenamento em condições ambiente apresentando médias de $14,5,14,9$ e $13,6 \mathrm{~N}$ respectivamente (Tabela 4 ).

Tabela 4. Valores médios sobre a firmeza dos frutos $(\mathrm{N})$ em graviolas armazenadas sob diferentes variações de gás carbônico $\left(\mathrm{CO}_{2}\right)$ em atmosfera controlada por quinze dias com posterior armazenamento em condições de temperatura ambiente por oito dias.

\begin{tabular}{lccc}
\hline \multirow{2}{*}{ Condições de Atmosfera Controlada } & \multicolumn{3}{c}{ Dias de armazenamento em temperatura ambiente } \\
\cline { 2 - 4 } & 0 (saída da câmara) & 4 & 8 \\
\hline & & Firmeza (N) & $14,5 \mathrm{aC}$ \\
$2,0 \mathrm{kPa} \mathrm{O}$ & $48,5 \mathrm{aA}$ & $30,0 \mathrm{aB}$ & $14,9 \mathrm{aC}$ \\
$2,0 \mathrm{kPa} \mathrm{O}+2,0 \mathrm{kPa} \mathrm{CO}_{2}$ & $49,2 \mathrm{aA}$ & $31,5 \mathrm{aB}$ & $13,6 \mathrm{aC}$ \\
$2,0 \mathrm{kPa} \mathrm{O}$ & $+2,5 \mathrm{kPa} \mathrm{CO}$ & $30,2 \mathrm{aB}$ & $6,54 \mathrm{bC}$ \\
$2,0 \mathrm{kPa} \mathrm{O}$ & $+3,0 \mathrm{kPa} \mathrm{CO}$ & $29,3 \mathrm{aB}$ & $6,21 \mathrm{bC}$ \\
\hline \multicolumn{1}{c}{$\mathrm{CV}=(\%)$} & $43,7 \mathrm{abA}$ & $29,8 \mathrm{aB}$ & 8,77 \\
\hline
\end{tabular}

Médias seguidas pela mesma letra na vertical não diferem entre si. Foi aplicado o teste de tukey ao nível de $5 \%$ de probabilidade. ns: não significativo.

Na Tabela 5 estão as médias para cada condição de AC sobre as variáveis acidez titulável, pH e sólidos solúveis sendo esta última a única que apresentou diferença estatística entre as diferentes pressões de $\mathrm{CO}_{2}$.

Os valores de acidez após oito dias de armazenamento em temperatura ambiente apresentaram resultados médios oscilando entre 0,79 a 0,86 g/100 g de ácido cítrico (Tabela 5).

Weber et al. (2013) e Both et al. (2014) também não observaram interação significativa para os valores médios de acidez titulável em maçãs sob diferentes condições de $A C$, ao passo que Corrêa et al. (2010) verificaram aumento nos valores de acidez quando mantidas em maiores pressões de $\mathrm{CO}_{2}$.

Os valores de $\mathrm{pH}$ permaneceram na faixa de 3,78a 3,83 bem inferior ao valor médio determinado antes do acondicionamento dos frutos nas condições de $A C$ de 5,36. Estes resultados estão de acordo com os apresentados por Lima et al. (2003) que em estudo com graviolas 
da variedade "Morada" após seis dias de armazenamento em temperatura ambiente identificaram valores variando entre 5,46 a 3,60 . As menores pressões de $\mathrm{CO}_{2}\left(2,0 \mathrm{kPa} \mathrm{O}{ }_{2}+<0,05 \mathrm{kPa}\right.$ $\mathrm{CO}_{2}$ e $\left.2,0 \mathrm{kPa} \mathrm{O}{ }_{2}+<1,0 \mathrm{kPa} \mathrm{CO}\right)_{2}$ ) apresentaram os maiores valores, contudo sem diferir estatisticamente dos demais (Tabela 5).

Os valores médios obtidos sobre o conteúdo diferiram apenas entre as variações de gás carbônico $\left(\mathrm{CO}_{2}\right)$, avaliada, não apresentando diferenças ao longo do tempo de armazenamento (Tabela 5). Após oito dias de armazenamento em condições ambiente os frutos sob as pressões de $2,0 \mathrm{kPaO}_{2}+2,0 \mathrm{kPaCO}$ e $2,0 \mathrm{kPaO} \mathrm{O}_{2}+3,0 \mathrm{kPa} \mathrm{CO}$ apresentaram as maiores médias 15,3 e $15,9^{\circ} \mathrm{Brix}$ respectivamente. As pressões de $2,0 \mathrm{kPaO} \mathrm{O}_{2}+<0,05$ $\mathrm{kPa} \mathrm{CO}_{2}$ e $2,0 \mathrm{kPa} \mathrm{O}+<1,0 \mathrm{kPa} \mathrm{CO}_{2}$ apresentaram valores para o mesmo período variando entre 12,8 e $12,3^{\circ}$ Brix, não diferindo entre si (Tabela 5).

Para Chitarra e Chitarra (2007), o aumento no teor de sólidos solúveis é esperado uma vez que o metabolismo respiratório das frutas e hortaliças degradam suas substâncias reservas como o amido e a pectina para produção de energia, deste modo, quanto maior o teor de sólidos, mais avançado encontra-se seu estádio de maturação.

Tabela 5. Valores médios sobre as variáveis sólidos solúveis, acidez titulável e pH em frutos de graviolas armazenadas sob diferentes variações de gás carbônico $\left(\mathrm{CO}_{2}\right)$ em atmosfera controlada por quinze dias com posterior acondicionamento em condições de temperatura ambiente por oito dias.

\begin{tabular}{|c|c|c|c|}
\hline Condições de Atmosfera Controlada & $\begin{array}{l}\text { Acidez titulável } \\
\text { (g/100g ácido } \\
\text { cítrico) }\end{array}$ & $\mathrm{pH}$ & $\begin{array}{c}\text { Sólidos solúveis } \\
\text { totais ( }{ }^{\circ} \text { Brix) }\end{array}$ \\
\hline Controle $\left.\left(2,0 \mathrm{kPa} \mathrm{O}{ }_{2}+<0,05 \mathrm{kPa} \mathrm{CO}\right)_{2}\right)$ & $0,79 \mathrm{~ns}$ & $3,83 \mathrm{~ns}$ & $12,8 \mathrm{a}$ \\
\hline $2,0 \mathrm{kPa} \mathrm{O}+<1,0 \mathrm{kPa} \mathrm{CO}$ & $0,82 \mathrm{~ns}$ & $3,81 \mathrm{~ns}$ & $12,3 \mathrm{a}$ \\
\hline $2,0 \mathrm{kPaO} \mathrm{O}_{2}+2,0 \mathrm{kPa} \mathrm{CO}$ & $0,81 \mathrm{~ns}$ & $3,79 \mathrm{~ns}$ & $13,5 a b$ \\
\hline $2,0 \mathrm{kPaO} \mathrm{O}_{2}+2,5 \mathrm{kPa} \mathrm{CO}$ & $0,86 \mathrm{~ns}$ & $3,78 \mathrm{~ns}$ & $15,3 \mathrm{~b}$ \\
\hline $2,0 \mathrm{kPaO} \mathrm{O}_{2}+3,0 \mathrm{kPa} \mathrm{CO}_{2}$ & $0,83 \mathrm{~ns}$ & $3,78 \mathrm{~ns}$ & $15,9 \mathrm{~b}$ \\
\hline $\mathrm{CV}=(\%)$ & 1,23 & 1,87 & 2,65 \\
\hline
\end{tabular}

Médias seguidas pela mesma letra na vertical diferem entre si. Foi aplicado o teste de tukey ao nível de $5 \%$ de probabilidade. ns: não significativo.

Para as variáveis, índice de iodo amido, incidência de podridões e degenerescência da polpa os dados não apresentaram diferenças ao longo do tempo de armazenamento quando expostos em temperatura ambiente (Tabela 6).

0 índice de amido de $10,86 \mathrm{~g} \cdot 100 \mathrm{~g}^{-1}$ apresentado pelos frutos antes da entrada nas câmaras decresceu significativamente apresentando valores após oito dias em condições ambiente de 2,56 e 2,64 para as condições de 2,0 $\mathrm{kPa}$ de $\mathrm{O}_{2}+<1,0 \mathrm{kPa}$ de $\mathrm{CO}_{2}$ e $2,0 \mathrm{kPa}$ de $\mathrm{O}_{2}+<0,05$ $\mathrm{kPa}$ de $\mathrm{CO}_{2}$, respectivamente (Tabela 6). As pressões de 2,0 e 3,0 de $\mathrm{kPa}$ de $\mathrm{CO}_{2}$ apresentaram para o mesmo período valores abaixo de 1,85 diferindo significativamente dos demais. A condição de $2,0 \mathrm{kPa}$ de $\mathrm{O}_{2}+2,0 \mathrm{kPa}$ de $\mathrm{CO}_{2}$ apresentou semelhança estatística quando comparada as baixas pressões de $\mathrm{CO}_{2}$ (Tabela 6).

Os valores apresentados neste experimento estão de acordo com os dados propostos por Lima et al. (2006) em trabalho com graviolas que encontraram variação média ao longo de seis dias em temperatura ambiente de 10,65 e $1,68 \mathrm{~g} / 100 \mathrm{~g}-1$.

Na saída da câmara não foram identificados indícios de podridões nos frutos independente da condição de AC. De acordo com Brackmann et al. (2003) o efeito na redução da ocorrência de podridões em atmosfera controlada é devido ao retardamento da degradação de pectinas da parede celular, tornando o fruto mais resistente à incidência de fungos.

Ao final de oito dias em temperatura ambiente o percentual de podridões ultrapassou $50 \%$ atingindo percentuais médios de 62,9 e $66,4 \%$ quando mantidos nas pressões maiores de $\mathrm{CO}_{2}(2,5$ e $3,0 \mathrm{kPa}$ de $\mathrm{CO} 2$ ) respectivamente, diferindo significativamente dos demais (Tabela 6) e concordando com o trabalho de Weber et al. (2013), onde o aumento das pressões parciais de 
$\mathrm{CO} 2$ de 2,0 para $2,5 \mathrm{kPa}$ favoreceu o aumento na incidência de podridões em maçãs.

A utilização das diferentes condições de AC não impediu a ocorrência de degenerescência da polpa após oito dias em temperatura ambiente verificando médias entre 19,8 e $21,9 \%$ sem diferença estatística (Tabela 6).

De modo geral nota-se que a baixa e a alta pressão de $\mathrm{O}_{2}$ e $\mathrm{CO}_{2}(2,0 \mathrm{kPa}$ de $\mathrm{O} 2+<0,05 \mathrm{kPa}$ de $\mathrm{CO} 2$ e $2,0 \mathrm{kPa} \mathrm{O}{ }_{2}+3,0 \mathrm{kPa} \mathrm{CO}_{2}$ ) resultaram nas maiores médias aos final do armazenamento, este fato pode estar ligado a falta de $\mathrm{O}_{2}$ na fase final de maturação quando o fruto necessita de mais oxigênio no processo respiratório, assim como o excesso de $\mathrm{CO}_{2}$ na fase final de maturação favorece a formação de injúrias no tecido dos frutos pela alta concentração de $\mathrm{CO}_{2}$ causando toxidez impulsionando o amadurecimento e consequentemente a degenerescência da polpa.

Tabela 6. Valores médios sobre as variáveis teor de amido, incidência de podridões e degenerescência da polpa em frutos de graviolas armazenadas sob diferentes variações de gás carbônico $\left(\mathrm{CO}_{2}\right)$ em atmosfera controlada por quinze dias com posterior acondicionamento em condições de temperatura ambiente por oito dias.

\begin{tabular}{|c|c|c|c|}
\hline Condições de Atmosfera Controlada & $\begin{array}{l}\text { Teor de amido } \\
\text { (g/100g-1) }\end{array}$ & $\begin{array}{l}\text { Incidência de } \\
\text { podridões (\%) }\end{array}$ & $\begin{array}{c}\text { Degenerescência da } \\
\text { polpa (\%) }\end{array}$ \\
\hline Controle $\left.\left(2,0 \mathrm{kPa} \mathrm{O}{ }_{2}+<0,05 \mathrm{kPa} \mathrm{CO}\right)_{2}\right)$ & $2,64 \mathrm{~b}$ & $53,2 \mathrm{a}$ & $21,9 \mathrm{~ns}$ \\
\hline $2,0 \mathrm{kPa} \mathrm{O}+<1,0 \mathrm{kPa} \mathrm{CO}$ & $2,56 \mathrm{~b}$ & $51,8 \mathrm{a}$ & $20,7 \mathrm{~ns}$ \\
\hline $2,0 \mathrm{kPaO}_{2}+2,0 \mathrm{kPa} \mathrm{CO} 2$ & $2,17 \mathrm{ab}$ & 54,6 a & $19,8 \mathrm{~ns}$ \\
\hline $2,0 \mathrm{kPaO}{ }_{2}+2,5 \mathrm{kPa} \mathrm{CO} 2$ & $1,81 \mathrm{a}$ & $62,9 \mathrm{~b}$ & $20,3 \mathrm{~ns}$ \\
\hline $2,0 \mathrm{kPa} \mathrm{O}+3,0 \mathrm{kPa} \mathrm{CO}$ & $1,78 \mathrm{a}$ & $66,4 \mathrm{~b}$ & $21,6 \mathrm{~ns}$ \\
\hline $\mathrm{CV}=(\%)$ & 1,56 & 3,54 & 6,39 \\
\hline
\end{tabular}

Médias seguidas pela mesma letra na vertical não diferem entre si. Foi aplicado o teste de tukey ao nível de $5 \%$ de probabilidade. ns: não significativo.

\section{CONCLUSÕES}

Altas pressões de $\mathrm{CO}_{2}\left(2,0 \mathrm{kPa} \mathrm{O} \mathrm{O}_{2}+3,0\right.$ $\mathrm{kPa} \mathrm{CO}_{2}$ e $2,0 \mathrm{kPa} \mathrm{O}_{2}+3,0 \mathrm{kPa} \mathrm{CO}_{2}$ ), não são indicadas para o armazenamento de graviolas variedade "Morada" pois sua ação favorece a perda da coloração da casca e da polpa dos frutos, acelera a síntese de etileno favorecendo a perda de qualidade após quatro dias em condições ambiente.

A baixa pressão de $\mathrm{CO}_{2}\left(2,0 \mathrm{kPa} \mathrm{O} \mathrm{O}_{2}+\right.$ $<0,05 \mathrm{kPa} \mathrm{CO}_{2}$ e $\left.2,0 \mathrm{kPa} \mathrm{O}+<1,0 \mathrm{kPa} \mathrm{CO}_{2}\right)$ garantiram os melhores resultados sobre as variáveis físico-químicas após quinze dias de acondicionamento mais oito dias em temperatura ambiente sendo, portanto, as pressões mais indicadas para o armazenamento das graviolas cultivar "Morada".

\section{REFERÊNCIAS}

AOAC. ASSOCIATION OF OFFICIAL ANALYTICAL CHEMISTRY. Official methods of analysis of the Association of Official Analytical Chemistry. 17. ed. Washington: AOAC, 2008. 1115p.
BOTH, V.; BRACKMANN, A.; WEBER, A.; ANESE, R. de. O.; THEWES, F. R. Estresse inicial por baixo oxigênio seguido do armazenamento em atmosfera controlada de maçãs 'Royal Gala'. Revista Ciência Agronômica, Fortaleza, v. 45, n. 4, p. 708-717, $2014 . \quad$ https://doi.org/10.1590/S180666902014000400008

BRACKMANN, A.; STEFFENS, C. A.; GIEHL, R. F. H. Armazenamento de pêssego 'Chimarrita' em atmosfera controlada e sob absorção de etileno. Ciência Rural, Santa Maria, v. 33, n. 3, p. 431-435, 2003. 84782003000300006

CHITARRA, M. I.; CHITARRA, A. B. Pós-colheita de frutos e hortaliças: fisiologia e manuseio. 2. ed. Lavras: UFLA, 2007. 785p.

CORRÊA, T.R.; STEFFENS, C. A.; AMARANTE, C. V. T.; BRACKMANN, A.; SILVEIRA, J. P. G.; TANAKA, H.; BOTH, V. Qualidade de maçãs 'Fuji' armazenadas em atmosfera controlada e influência do clima na degenerescência da polpa. Pesquisa Agropecuária Brasileira, Brasília, v.45, n.6, p. 531-538, 2010. https://doi.org/10.1590/S0100-

204X2010000600001 
CUNHA JUNIOR, L. C.; JACOMINO, A. P.; OGASSAVARA, F. O.; TREVISAN, M. J.; PARISI, M. C. $M$. Armazenamento refrigerado de morando submetido a altas concentrações de $\mathrm{CO}_{2}$. Revista Horticultura Brasileira, Brasília, v. 30, n. 4. p. 688694, 2012. https://doi.org/10.1590/S010205362012000400020

FRANCK, C.; LAMMERTYN, J.; HO, Q. T.; VERBOVEN, P.; NICOLAI, B. M. Browning disorders in pear fruit. Postharvest Biology and Technology, Amsterdam, v.43, n.1, p.1-13, 2007. https://doi.org/10.1016/j.postharvbio.2006.08.008 FREITAS, A. L. G. E.; VILAS BOAS, F. S.; PIRES, M. M.; SÃO JOSÉ, A. R. caracterização da produção e do mercado da graviola (Annona muricata I.) no estado da Bahia. Informações Econômicas, São Paulo, v. 43, n. 3, p. 86-95, 2013.

GUPTA, K. J.; ZABALZA, A.; VAN DONGEN, J. T. Regulation of respiration when the oxygen availability changes. Physiologia Plantarum, Copenhagem, v. 137, n. 4, p. 383-391, 2009.

LIMA, M. A. C.; ALVES, R. E.; FILGUEIRAS, H. A. C.; ENÉAS-FILHO, J. Comportamento respiratório e qualidade pós-colheita de graviola (Annona muricata L.) 'morada' sob temperatura ambiente. Revista Brasileira de Fruticultura. Jaboticabal-SP, v. 25, n. 1, p. 49-52, 2003.

LIMA, M. A. C.; ALVES, R. E.; FILGUEIRAS, H. A. C.; ENÉAS-FILHO, J. Mudanças relacionadas ao amaciamento da graviola durante a maturação póscolheita. Pesquisa Agropecuária Brasileira., Brasília-DF, v. 41, n. 12, p. 1707-1713, 2006.

PAYASI, A. MISHRA, N.N.; CHAVES, A. L. S.; SINGH, R. Biochemistry of fruit softening: an overview. Physiology and Molecular Biology of Plants, Springer, v.15, n.2, p.103-113, 2009. https://doi.org/10.1007/s12298-009-0012-z

PEDRESCHI, R.; FRANCK, C.; LAMMERTYN, J.; ERBAN, A.; KOPKA, J.; HERTOG, M.; VERLINDEN, B.; NICOLAI, B. Metabolic profiling of Conference pears under low oxygen stress. Postharvest Biology and Technology, v.51, p.123-130, 2009. https://doi.org/10.1016/j.postharvbio.2008.05.019 STEFFENS, C. A.; TANAKA, H.; AMARANTE, C. V. T. do.; BRACKMANN, A.; STANGER, M. C.; HENDGES, M. V. Condições de atmosfera controlada para armazenamento de ameixas "Laetitia" tratadas com 1-meticiclopropeno. Revista Ciência Agronômica, Fortaleza, v.44, n.4. p.750-756, 2013.
https://doi.org/10.1590/S180666902013000400011

SUGAR, D.; BASILE, S.R. Integrated ethylene and temperature conditioning for induction of ripening capacity in 'Anjou' and 'Comice' pears. Postharvest Biology and Technology, Amsterdam, v.83, n.2, p.9-16, 2013. https://doi.org/10.1016/j.postharvbio.2013.03.010 WEBER, A. BRACKMANN, A.; ANESE, R. O.; BOTH, V. PAVANELLO, E. P. Atmosfera controlada para o armazenamento da maçã 'Maxi Gala'. Revista Ciência Agronômica, Fortaleza, v. 44, n. 2, p. 294301 , 2013. https://doi.org/10.1590/S180666902013000200011

ZACARONI, A. B.; JUNQUEIRA, N. T. V.; SUSSEL, A. A. A. B.; FREITAS, I. S., BRAGA, M. F., JUNQUEIRA, K. P. Desempenho agronômico de gravioleira (Annona muricata L.) sobre diferentes espécies de portaenxertos. Cadernos de Agroecologia, Porto Alegre, v. 9, n. 3 , p. $116-120,2014$.

Recebido para publicação em 21/05/2016

Revisado em 17/11/2016

Aceito em 06/02/2017 